\title{
A RECURRENCE THEOREM ON THE SOLUTIONS TO THE 2D EULER EQUATION*
}

\author{
Y. CHARLES $\mathrm{LI}^{\dagger}$
}

\begin{abstract}
In this article, I will prove a recurrence theorem which says that any $H^{s}\left(\mathbb{T}^{2}\right)(s>2)$ solution to the 2D Euler equation returns repeatedly to an arbitrarily small $H^{0}\left(\mathbb{T}^{2}\right)$ neighborhood.

Key words. Poincaré recurrence, 2D Euler equation, kinetic energy, enstrophy, compact embedding.
\end{abstract}

AMS subject classifications. Primary 37, 76; Secondary 35, 34

1. Introduction. In finite dimensions, the Poincaré recurrence theorem can be proved from the basic properties of a finite measure. In infinite dimensions, it is difficult to define a physically meaningful finite measure [8]. It turns out that one can study the Poincaré recurrence problem using Banach norm. An interesting problem is to study the Poincaré recurrence problem for 2D Euler equation of fluids [6]. Nadirashvili [7] gave an example of Poincaré non-recurrence near a particular solution of the 2D Euler equation defined on an annular domain. The proof in [7] was fixed up in [6]. We believe that the Poincaré recurrence will occur more often when the 2D Euler equation is defined on a periodic domain. The theorem to be proved in this article is a result along this line. On a periodic domain, the 2D Euler equation is a more natural Hamiltonian system than e.g. on an annular domain.

One final note is that any solution of the 2D Euler equation defines a nonautonomous integrable Hamiltonian two dimensional vector field [5]. The trajectories of this vector field are the fluid particle trajectories. This is the so-called Lagrangian coordinates. The integrability was proved in the usual extended coordinates of two spatial coordinates, the stream function, and an extra temporal variable. Due to the extra temporal variable, most of the invariant subsets are of infinite volume. Only in special cases e.g. the solution of the 2D Euler equation is periodic or quasi-periodic in time, one can find invariant subsets of finite volume. This indicates that in the Lagrangian coordinates, recurrence is a rare event [9].

There have been a lot of studies on Euler equations from different perspectives [3] [10] [1] [11] [2]. Here we study their Poincaré recurrence problem. The proof of our theorem depends upon the fact that both kinetic energy and enstrophy are conserved for the 2D Euler equations. Therefore, our proof does not work for 3D Euler equations.

2. Finite Dimensional System. In finite dimensions, our theorem is a result of a simple compactness argument. But it states some interesting fact. In infinite dimensions, the compactness argument is more complicated.

TheOREm 2.1. Let $f: \mathbb{R}^{n} \mapsto \mathbb{R}^{n}$ be a map and $A$ be a compact invariant subset. Then for any $x \in A$ and any $\delta>0$, there is a $x_{*} \in A$ such that

$$
f^{m_{j}}(x) \in B_{\delta}\left(x_{*}\right)
$$

\footnotetext{
*Received October 3, 2007; accepted for publication July 11, 2008.

${ }^{\dagger}$ Department of Mathematics, University of Missouri, Columbia, MO 65211, USA (cli@math. missouri.edu).
} 
where $\left\{m_{j}\right\}$ is an infinite sequence of positive integers and $B_{\delta}\left(x_{*}\right)$ is the open ball of radius $\delta$ centered at $x_{*}$.

Proof. It is clear that $\left\{B_{\delta}(y)\right\}_{y \in A}$ is an open cover of $A$, thus there is a finite subcover $\left\{B_{\delta}\left(y_{k}\right)\right\}_{k=1, \cdots, K}$. For any $m=0,1,2, \cdots ; f^{m}(x) \in A$; thus $f^{m}(x) \in B_{\delta}\left(y_{k}\right)$ for some $k$. Therefore, there is at least one $k$ such that an infinite subsequence of $\left\{f^{m}(x)\right\}$ is included in $B_{\delta}\left(y_{k}\right)$. The theorem is proved.

REMARK 2.2. Of course the theorem is still true when replacing $\mathbb{R}^{n}$ by a Banach space or a topological space. But compactness is a very restricted concept in infinite dimensions.

3. 2D Euler Equation. The 2D Euler equation

$$
\partial_{t} u+(u \cdot \nabla) u=-\nabla p, \quad \nabla \cdot u=0
$$

is globally well-posed in $H^{s}\left(\mathbb{T}^{2}\right)(s>2)$ where $\mathbb{T}^{2}$ is the 2 -torus. We also require that

$$
\int_{\mathbb{T}^{2}} u d x=0
$$

Denote by $\omega$ the vorticity, $\omega=\partial_{1} u_{2}-\partial_{2} u_{1}$. A well-known fact is the following lemma.

LEMMA 3.1.

$$
\int_{\mathbb{T}^{2}}|\nabla u|^{2} d x=\int_{\mathbb{T}^{2}} \omega^{2} d x
$$

Proof. By the incompressibility condition,

$$
\int_{\mathbb{T}^{2}}\left[\left(\partial_{1} u_{1}\right)^{2}+\left(\partial_{2} u_{2}\right)^{2}\right] d x=-2 \int_{\mathbb{T}^{2}}\left(\partial_{1} u_{1}\right)\left(\partial_{2} u_{2}\right) d x .
$$

Since

$$
\begin{aligned}
& \int_{\mathbb{T}^{2}}\left[\left(\partial_{1} u_{1}\right)\left(\partial_{2} u_{2}\right)-\left(\partial_{2} u_{1}\right)\left(\partial_{1} u_{2}\right)\right] d x \\
& =\int_{\mathbb{T}^{2}}\left[\partial_{1}\left(u_{1} \partial_{2} u_{2}\right)-\partial_{2}\left(u_{1} \partial_{1} u_{2}\right)\right] d x=0,
\end{aligned}
$$

we have

$$
\begin{aligned}
\int_{\mathbb{T}^{2}}|\nabla u|^{2} d x & =\int_{\mathbb{T}^{2}}\left[\left(\partial_{1} u_{1}\right)^{2}+\left(\partial_{2} u_{1}\right)^{2}+\left(\partial_{1} u_{2}\right)^{2}+\left(\partial_{2} u_{2}\right)^{2}\right] d x \\
& =\int_{\mathbb{T}^{2}}\left[\left(\partial_{2} u_{1}\right)^{2}+\left(\partial_{1} u_{2}\right)^{2}-2\left(\partial_{1} u_{1}\right)\left(\partial_{2} u_{2}\right)\right] d x \\
& =\int_{\mathbb{T}^{2}}\left[\left(\partial_{2} u_{1}\right)^{2}+\left(\partial_{1} u_{2}\right)^{2}-2\left(\partial_{2} u_{1}\right)\left(\partial_{1} u_{2}\right)\right] d x \\
& =\int_{\mathbb{T}^{2}} \omega^{2} d x
\end{aligned}
$$


REMARK 3.2. Using Fourier series, one can prove this lemma by direct calculation:

$$
\begin{aligned}
u(k) & =\left(i k_{2},-i k_{1}\right) \frac{1}{|k|^{2}} \omega(k), \\
\int_{\mathbb{T}^{2}}|\nabla u|^{2} d x & =\sum_{k \in \mathbb{Z}^{2} /\{0\}}\left[k_{1}^{2} k_{2}^{2}+k_{2}^{4}+k_{1}^{4}+k_{1}^{2} k_{2}^{2}\right] \frac{1}{|k|^{4}} \omega(k)^{2} \\
& =\sum_{k \in \mathbb{Z}^{2} /\{0\}} \omega(k)^{2}=\int_{\mathbb{T}^{2}} \omega^{2} d x . \quad
\end{aligned}
$$

Notice that the kinetic energy

$$
E=\int_{\mathbb{T}^{2}}|u|^{2} d x
$$

and the enstrophy

$$
G=\int_{\mathbb{T}^{2}} \omega^{2} d x
$$

are two invariants of the 2D Euler flow.

Lemma 3.3. For any $C>0$, the set

$$
S=\left\{u \mid \int_{\mathbb{T}^{2}} \omega^{2} d x \leq C\right\}
$$

is compactly embedded in $L^{2}\left(\mathbb{T}^{2}\right)$ of $u$. That is, the closure of $S$ in $L^{2}\left(\mathbb{T}^{2}\right)$ is a compact subset of $L^{2}\left(\mathbb{T}^{2}\right)$.

REMARK 3.4. In a simpler language, an enstrophy ball is compactly embedded in the kinetic energy space. This lemma is the well-known Rellich lemma. In the $\mathbb{T}^{2}$ setting, we will present the proof. There are many versions of the lemma and its proof. We follow that of [4].

Proof. By Lemma 3.1,

$$
\int_{\mathbb{T}^{2}}|\nabla u|^{2} d x \leq C
$$

Let $\left\{u^{(j)}\right\}$ be a sequence in $S$. For any $\epsilon>0$, choose $K>0$ such that

$$
2 K^{-2} C<\epsilon / 2 \text {. }
$$

For any $k \in \mathbb{Z}^{2} /\{0\},|k| \leq K$, the Fourier coefficients $\left\{u^{(j)}(k)\right\}$ form a bounded set (e.g. bounded by $\sqrt{C}$ ). Therefore there is a convergent subsequence $\left\{u^{\left(m_{j}\right)}(k)\right\}$. For a different $\tilde{k},\left\{u^{\left(m_{j}\right)}(\tilde{k})\right\}$ is a bounded set again, thus there is a further convergent subsequence. Iterating on all such $k(|k| \leq K$ finitely many), one can find a subsequence $\left\{u^{\left(n_{j}\right)}\right\}$ such that $\left\{u^{\left(n_{j}\right)}(k)\right\}$ is uniformly convergent for $|k| \leq K$. This is 
a simpler version of the usual diagonal argument. Next we show that $\left\{u^{\left(n_{j}\right)}\right\}$ is a Cauchy sequence in $L^{2}\left(\mathbb{T}^{2}\right)$.

$$
\begin{aligned}
\left\|u^{\left(n_{j}\right)}-u^{\left(n_{\ell}\right)}\right\|_{L^{2}\left(\mathbb{T}^{2}\right)}= & \sum_{|k| \leq K, k \neq 0}\left|u^{\left(n_{j}\right)}(k)-u^{\left(n_{\ell}\right)}(k)\right|^{2} \\
& +\sum_{|k|>K}\left|u^{\left(n_{j}\right)}(k)-u^{\left(n_{\ell}\right)}(k)\right|^{2} \\
\leq & \sum_{|k| \leq K, k \neq 0}\left|u^{\left(n_{j}\right)}(k)-u^{\left(n_{\ell}\right)}(k)\right|^{2} \\
& +K^{-2} \sum_{|k|>K}|k|^{2}\left|u^{\left(n_{j}\right)}(k)-u^{\left(n_{\ell}\right)}(k)\right|^{2} .
\end{aligned}
$$

The second term is less than $2 K^{-2} C<\epsilon / 2$. The first term is less than $\epsilon / 2$ when $j$ and $\ell$ are sufficiently large since $\left\{u^{\left(n_{j}\right)}(k)\right\}$ is uniformly convergent for $|k| \leq K$. So $\left\{u^{\left(n_{j}\right)}\right\}$ is a Cauchy sequence in $L^{2}\left(\mathbb{T}^{2}\right)$, and is convergent inside the closure of $S$ in $L^{2}\left(\mathbb{T}^{2}\right)$. Let $\left\{v^{(j)}\right\}$ be a sequence of the accumulation points of $S$ in $L^{2}\left(\mathbb{T}^{2}\right)$. Then we can find a sequence $\left\{u^{(j)}\right\}$ in $S$ such that

$$
\left\|v^{(j)}-u^{(j)}\right\|_{L^{2}\left(\mathbb{T}^{2}\right)}<1 / j .
$$

Let $\left\{u^{\left(n_{j}\right)}\right\}$ be the convergent subsequence, then $\left\{v^{\left(n_{j}\right)}\right\}$ is also a convergent subsequence. Thus the closure of $S$ in $L^{2}\left(\mathbb{T}^{2}\right)$ is a compact subset of $L^{2}\left(\mathbb{T}^{2}\right)$.

THEOREM 3.5. For any $\tilde{u} \in H^{s}\left(\mathbb{T}^{2}\right)(s>2)$, any $\delta>0$, and any $T>0$; there is a $u^{*} \in H^{s}\left(\mathbb{T}^{2}\right)$ such that

$$
F^{m_{j} T}(\tilde{u}) \in B_{\delta}^{0}\left(u^{*}\right)=\left\{\hat{u} \in H^{s}\left(\mathbb{T}^{2}\right) \mid\left\|\hat{u}-u^{*}\right\|_{H^{0}\left(\mathbb{T}^{2}\right)}<\delta\right\}
$$

where $\left\{m_{j}\right\}$ is an infinite sequence of positive integers, and $F^{t}$ is the evolution operator of the $2 D$ Euler equation.

Proof. Choose the $C$ in Lemma 3.3 to be

$$
2 \int_{\mathbb{T}^{2}}|\nabla \tilde{u}|^{2} d x=2 \int_{\mathbb{T}^{2}} \tilde{\omega}^{2} d x .
$$

Define two sets:

$$
\begin{aligned}
S & =\left\{u \mid \int_{\mathbb{T}^{2}} \omega^{2} d x \leq 2 \int_{\mathbb{T}^{2}} \tilde{\omega}^{2} d x\right\}, \\
S_{1} & =\left\{u \in H^{s}\left(\mathbb{T}^{2}\right) \mid \int_{\mathbb{T}^{2}} \omega^{2} d x \leq 2 \int_{\mathbb{T}^{2}} \tilde{\omega}^{2} d x\right\} .
\end{aligned}
$$

Notice that $S_{1}$ is invariant under the 2D Euler flow, and $S_{1}$ is a dense subset of $S$, $S_{1}=S \cap H^{s}\left(\mathbb{T}^{2}\right)$. By Lemma 3.3, the closure of $S$ in $L^{2}\left(\mathbb{T}^{2}\right)=H^{0}\left(\mathbb{T}^{2}\right)$ is a compact subset. For any $u \in S$, denote by

$$
B_{\delta / 2}(u)=\left\{u \in H^{0}\left(\mathbb{T}^{2}\right) \mid\|v-u\|_{H^{0}\left(\mathbb{T}^{2}\right)}<\delta / 2\right\} .
$$

All these balls $\left\{B_{\delta / 2}(u)\right\}_{u \in S}$ is an open cover of the closure of $S$ in $H^{0}\left(\mathbb{T}^{2}\right)$. Thus there is a finite subset $\left\{u_{1}, \cdots, u_{N}\right\} \subset S$ such that $\left\{B_{\delta / 2}\left(u_{n}\right)\right\}_{n=1, \cdots, N}$ is a finite cover. Since $S_{1}$ is dense in $S$, for each such $u_{n}$, one can find a $u_{n}^{*} \in S_{1}$ such that

$$
\left\|u_{n}-u_{n}^{*}\right\|_{H^{0}\left(\mathbb{T}^{2}\right)} \leq\left\|u_{n}-u_{n}^{*}\right\|_{W^{1,2}\left(\mathbb{T}^{2}\right)}<\delta / 4
$$


by the Poincaré inequality and the mean zero condition $(3.1)$, where $W^{1,2}\left(\mathbb{T}^{2}\right)$ is the Sobolev space with norm $\|u\|^{2}=\int_{\mathbb{T}^{2}}|\nabla u|^{2} d x$. By Lemma 3.1, for our mean-zero divergence-free velocity field, $\int_{\mathbb{T}^{2}}|\nabla u|^{2} d x=\int_{\mathbb{T}^{2}} \omega^{2} d x$. All the balls

$$
B_{\delta}\left(u_{n}^{*}\right)=\left\{v \in H^{0}\left(\mathbb{T}^{2}\right) \mid\left\|v-u_{n}^{*}\right\|_{H^{0}\left(\mathbb{T}^{2}\right)}<\delta\right\}
$$

still covers $S$, thus covers $S_{1}=S \cap H^{s}\left(\mathbb{T}^{2}\right)$. Let $B_{\delta}^{0}\left(u_{n}^{*}\right)=B_{\delta}\left(u_{n}^{*}\right) \cap H^{s}\left(\mathbb{T}^{2}\right)$,

$$
B_{\delta}^{0}\left(u_{n}^{*}\right)=\left\{\hat{u} \in H^{s}\left(\mathbb{T}^{2}\right) \mid\left\|\hat{u}-u_{n}^{*}\right\|_{H^{0}\left(\mathbb{T}^{2}\right)}<\delta\right\} .
$$

Then

$$
S_{1} \subset \bigcup_{n=1}^{N} B_{\delta}^{0}\left(u_{n}^{*}\right) .
$$

By the invariance of $S_{1}$ under the 2D Euler flow $F^{t}$, there is at least one $n$ such that an infinite subsequence of $\left\{F^{m T}(\tilde{u})\right\}_{m=0,1, \ldots}$ is included in $B_{\delta}^{0}\left(u_{n}^{*}\right)$. The theorem is proved. $\mathrm{Q}$

\section{REFERENCES}

[1] P. Constantin, On the Euler equations of incompressible fluids, Bull. AMS, 44 (2007), pp. 603621.

[2] C. Doering and J. Gibbon, Applied Analysis of the Navier-Stokes Equations, Cambridge Univ. Press, 1995.

[3] G. Eyink and K. Sreenivasan, Onsager and the theory of hydrodynamic turbulence, Rev. Mod. Phys., 78 (2006), pp. 87-135.

[4] G. Folland, Introduction to Partial Differential Equations, Princeton University Press, (1976), pp. 253-255.

[5] Y. Lan And Y. Li, On the dynamics of Navier-Stokes and Euler equations, J. Stat. Phys., 132 (2008), pp. 35-76, Appendix B.

[6] Y. LI, The Poincaré recurrence problem of inviscid incompressible fluids, the same issue, (2008) also available at: http://www.math.missouri.edu/ ${ }^{\sim}$ cli/Recurrence1.pdf.

[7] N. Nadirashvili, Wandering solutions of Euler's D-2 equation, Funct. Anal. Appl., 25:3 (1991), pp. 220-221.

[8] W. Ott and J. Yorke, Prevalence, Bull. AMS, 42:3 (2005), pp. 263-290.

[9] A. Shnirelman, Evolution of singularities, generalized Liapunov function and generalized integral for an ideal incompressible fluid, Amer. J. Math., 119 (1997), pp. 579-608.

[10] A. Shnirelman, On the nonuniqueness of weak solution of the Euler equation, Comm. Pure Appl. Math., 50:12 (1997), pp. 1261-1286.

[11] R. Temam, Infinite Dimensional Dynamical Systems in Mechanics and Physics, Springer, Appl. Math. Sci. Ser., 68, 1988. 
Y. C. LI 